\title{
Effect of metformin on bioactive lipid metabolism in insulin-resistant muscle
}

\author{
Piotr Zabielski,2, Marta Chacinska2,3, Karol Charkiewicz',4, Marcin Baranowski2, \\ Jan Gorski² and Agnieszka U Blachnio-Zabielska ${ }^{2,3}$ \\ 1Department of Medical Biology, Medical University of Bialystok, Bialystok, Poland \\ 2Department of Physiology, Medical University of Bialystok, Bialystok, Poland \\ ${ }^{3}$ Department of Hygiene, Epidemiology and Metabolic Disorders, Medical University of Bialystok, \\ Bialystok, Poland \\ ${ }^{4}$ Department of Perinatology, Medical University of Bialystok, Bialystok, Poland
}

Correspondence should be addressed to A U Blachnio-Zabielska Email

agnieszka.blachnio@umb. edu.pl

\begin{abstract}
Intramuscular accumulation of bioactive lipids leads to insulin resistance and type 2 diabetes (T2D). There is lack of consensus concerning which of the lipid mediators has the greatest impact on muscle insulin action in vivo. Our aim was to elucidate the effects of high-fat diet (HFD) and metformin (Met) on skeletal muscle bioactive lipid accumulation and insulin resistance (IR) in rats. We employed a [U-13C]palmitate isotope tracer and mass spectrometry to measure the content and fractional synthesis rate (FSR) of intramuscular long-chain acyl-COA (LCACOA), diacylglycerols (DAG) and ceramide (Cer). Eight weeks of HFD-induced intramuscular accumulation of LCACoA, DAG and Cer accompanied by both systemic and skeletal muscle IR. Metformin treatment improved insulin sensitivity at both systemic and muscular level by the augmentation of Akt/PKB and AS160 phosphorylation and decreased the content of DAG and Cer and their respective FSR. Principal component analysis (PCA) of lipid variables revealed that altered skeletal muscle IR was associated with lipid species containing 18-carbon acyl-chain, especially with C18:0-Cer, C18:1-Cer, 18:0/18:2-DAG and 18:2/18:2-DAG, but not palmitatederived lipids. It is concluded that the insulin-sensitizing action of metformin in skeletal muscle is associated with decreased 18-carbon acyl-chain-derived bioactive lipids.
\end{abstract}

\section{Key Words}

- type 2 diabetes

- high-fat diet

- insulin resistance

- metformin

- ceramide

- diacylglycerol

- skeletal muscle

\section{Introduction}

Consumption of high-fat diet (HFD) and obesity are associated with accumulation of intramuscular lipids, leading to insulin resistance (IR) and type 2 diabetes (T2D). The mechanism underlying the induction of IR is still incompletely understood. Skeletal muscle is responsible for $70-80 \%$ of whole body insulin-stimulated glucose uptake and plays an important role in lipid metabolism. In obese and type 2 diabetic subjects, plasma free fatty acids (FFA) are often elevated compared to lean, healthy individuals (Belfort et al. 2005). Increased fatty acid uptake was observed in skeletal muscle from subjects consuming HFD (Roepstorff et al. 2004), individuals with T2D (Bruce et al. 2003) and obese humans (Simoneau et al. 1999). After entering the cell, FA are activated to longchain fatty acyl-CoA (LCACoA), which are channeled toward mitochondrial $\beta$-oxidation through conversion to long-chain acyl-carnitines (LCAC) or de novo synthesis of intramyocellular lipids (IMCL) (Jeukendrup 2002). It is suggested that excess of LCACoA leads to overload of mitochondrial oxidative capacity and accumulation of IMCLs. This mechanism is amplified by the accumulation of malonyl-CoA which inhibits the activity of carnitine 
palmitoyltransferase (CPT-1) and diverts LCACoA toward de novo synthesis of lipids. The increased intracellular FA uptake and decline in mitochondrial FA channeling likely contributes to accumulation of IMCL and induction of IR. Current data suggest that intramuscular LCACoAs, diacylglycerols (DAG) and ceramides (Cer) promote the onset on IR by inhibiting key proteins within the insulin signaling pathway (Fig. 1). There is an ongoing discussion about which molecular lipid species play the leading role in FA-induced skeletal muscle IR. Elevated LCACoAs and impaired insulin-stimulated glucose uptake have been reported in skeletal muscle of obese individuals (Hulver et al. 2003) and with consumption of HFD in skeletal muscle of rats (Ellis et al. 2000). The accumulation of intracellular DAG has been demonstrated in the insulin-resistant muscle of HFD rats (Schmitz-Peiffer et al. 1997) and Zucker rats (Turinsky et al. 1990), whereas Cer accumulation has been shown in the muscles of obese, insulin-resistant rats (Turinsky et al. 1990) and obese insulin-resistant humans (Adams et al. 2004). The accumulation of LCACoAs and DAGs may interfere with insulin signaling through the activation of certain serine/ threonine PKC isoenzymes (Itani et al. 2002), whereas accumulation of Cer can inhibit the insulin signaling pathway via protein phosphatase 2A (PPA2)-mediated inhibition of Akt/PKB (Schmitz-Peiffer et al. 1999) (Fig. 1).

Currently, there is a shift in interest from total intramyocellular fat accumulation to bioactive lipid classes and individual lipid species in the pathophysiology of insulin resistance (Kitessa \& Abeywardena 2016). It appears that the acyl-chain length of particular bioactive lipids is an important factor in their deleterious effects on insulin signaling. The use of stable isotope-labeled FA and mass spectrometry allows for establishing the fate of intracellular fatty acids and precise measurement of fractional synthesis rate (FSR) of particular lipid species derived from labeled FA. Intramuscular content of bioactive lipids depends on the balance between lipid synthesis and degradation. For example, Cer accumulation can originate from de novo synthesis or release from complex sphingolipids such as sphingomyelins, glucosyl and galactosyl ceramides. Conversely, reduction in intramuscular Cer can arise from its hydrolysis or incorporation into complex sphingolipids. The use of stable isotope tracers allows for the identification of particular mechanism. Increase in the label incorporation without change in lipid concentration suggests increased incorporation of the lipid into complex species, whereas its accumulation under decreased labeling points to the degradation of complex species. Therefore, the main goal of this study was to measure both the concentration and synthesis rate (FSR) of different palmitate-derived lipid intermediates (LCACoAs, LCACs, DAGs, Cers) together with the expression of associated enzymes and correlate lipid variables with skeletal muscle IR in HFDfed rats. We used the well-defined experimental model of HFD-induced skeletal muscle IR in rat and employed commonly prescribed insulin sensitizer, metformin, to reverse diet-induced insulin resistance. As palmitate (a major plasma FA) is widely used in studies of lipid-induced IR and serves as crucial intermediate in Cer synthesis, we employed a stable isotope-labeled $\left[\mathrm{U}-{ }^{13} \mathrm{C}\right]$ palmitate

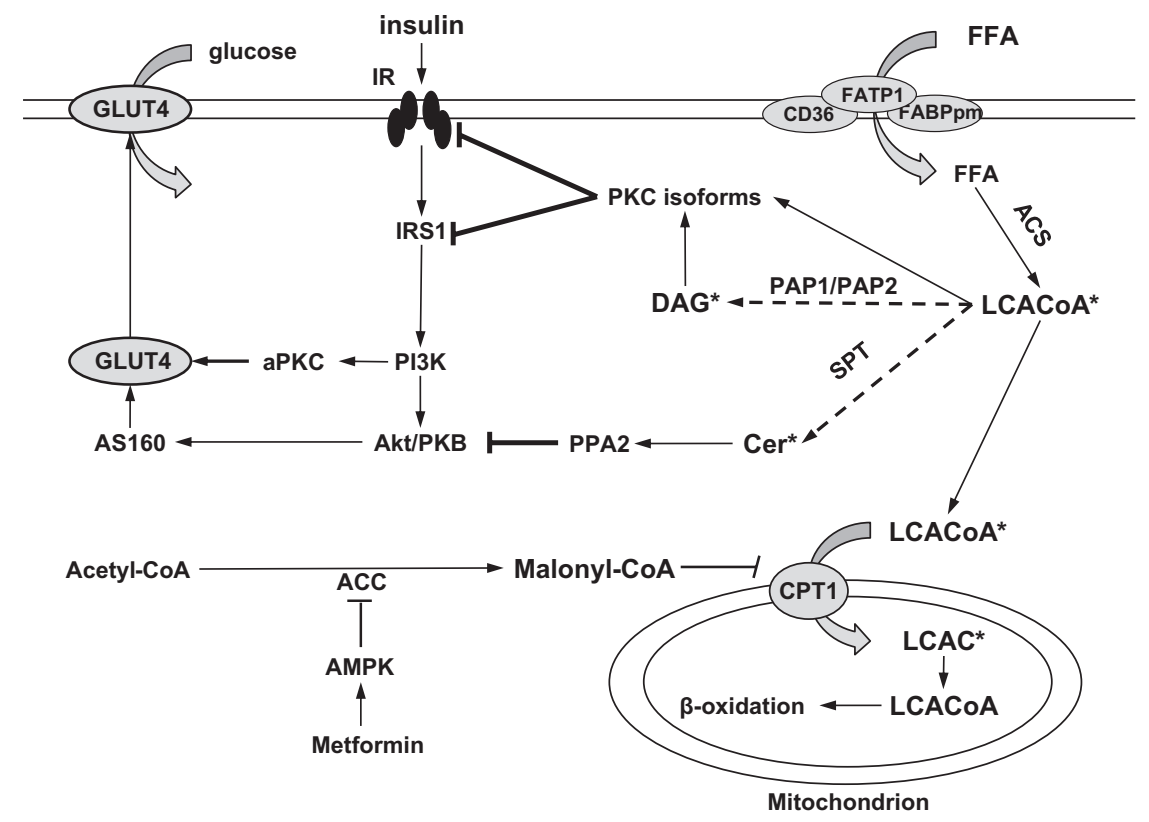

Figure 1

Mechanism of FFA-induced inhibition of insulin signaling. CD36, fatty acid translocase; FATP1, fatty acid transport protein 1; FABPpm, fatty acid-binding protein (plasma membrane); ACS, acyl-coenzyme A synthetase; LCACoA, long-chain acyl-CoAs; LCAC, long-chain acyl-carnitines; SPT, serine palmitoyltransferase; PPA2, protein phosphatase A2; PAP1/PAP2, phosphatidate phosphatase, isoforms 1 and 2; Akt/PKB, protein kinase $B ;$ DAG, diacylglycerol; $P K C$, protein kinase $C_{;} I R$, insulin receptor; IRS1, insulin receptor substrate 1 ; GLUT4, glucotransporter 4; AS160, Akt substrate 160; PI3K, phosphatidylinositol-3-kinase; aPKC, atypical protein kinase C; ACC, acetyl-CoA carboxylase; AMPK, AMP-activated kinase; CPT1, carnitine palmitoyltransferase 1 . *Denotes lipids with measured concentration and FSR. 
tracer. Together with ultra-high-performance liquid chromatography (UHPLC)-tandem mass spectrometry (MS/MS), we measured FSR of palmitate-derived bioactive lipids in dietary-induced IR. To the best of our knowledge, our work is the first to describe the FSR of 16:0-CoA, 16:0AC, 16:0-Cer, 16:0/16:0-DAG and C16:0/C18:1-DAG in complex experimental setup. Moreover, by applying metformin in HFD-induced insulin-resistant rats, together with an ability of measuring FSR of palmitatederived lipids, we were able to establish the impact of metformin on the accumulation and synthesis rate of particular lipid species.

\section{Materials and methods}

\section{Animals and study design}

All procedures were approved by the Institutional Animal Care and Use Committee of Medical University of Bialystok. The experiments were carried out on male Wistar rats ( 6 weeks of initial age, $150 \mathrm{~g}$ of initial body weight). Animals were divided into following groups: (1) Control, fed control diet for 8 weeks (Research Diets INC D12450B, $10 \%$ calories from fat) $(n=8)$; (2) high-fat diet (HFD), fed high-fat diet for 8 weeks (Research Diets INC D12492, $60 \%$ calories from saturated fat) $(n=8)$; (3) metformintreated (HFD/Met) fed HFD for 8 weeks and treated with metformin by oral gavage $(300 \mathrm{mg} / \mathrm{kg})$, starting from 5 th week of HFD feeding $(n=8)$. One week before euthanasia, an oral glucose tolerance test (OGTT) was performed. After 3 days of recovery, animals were subjected to intraperitoneal insulin tolerance test (IPTT). On the last day of the study, the food was withdrawn at 06:00 $\mathrm{h}$ and animals were fasted for $6 \mathrm{~h}$. The [U-13C]palmitate tracer was given for $2 \mathrm{~h}$ as primed continuous infusion $(0.5 \mu \mathrm{mol}$ bolus; $0.3 \mu \mathrm{mol} / \mathrm{kg} / \mathrm{h}$ continuous) through catheter placed in lateral tail vain. During the last $30 \mathrm{~min}$, insulin bolus $(0.5 \mathrm{U} / \mathrm{kg})$ was administrated intravenously to measure insulin-stimulated protein phosphorylation. The rats were anesthetized by intraperitoneal injection of pentobarbital at a dose of $80 \mathrm{mg} / \mathrm{kg}$. Both soleus muscles were removed, placed on ice and cleaned of connective tissue and fat. Tissue was frozen in liquid nitrogen and stored at $-80^{\circ} \mathrm{C}$ until analysis.

\section{Tracer infusion}

Albumin-bound $\left[\mathrm{U}-{ }^{13} \mathrm{C}\right]$ palmitate (potassium salt, uniformly labeled, Sigma-Aldrich) was prepared as previously described (Guo et al. 1997). Plasma palmitate concentration and isotopic enrichment were measured according to Persson and coworkers (Persson et al. 2010). Isotope tracer infusion was performed according to Blachnio-Zabielska and coworkers (BlachnioZabielska et al. 2013). The infusion protocol resulted in stable isotopic enrichment of plasma palmitate during $2 \mathrm{~h}$ of tracer infusion (Supplementary Fig. 1A, see section on supplementary data given at the end of this article). The area under palmitate enrichment curve was identical in all experimental groups (Supplementary Fig. 1B). 'Extended materials and methods' section of the supplement contains detailed description of tracer preparation, infusion and calculation of lipid turnover and FSR.

\section{Measurement of lipid concentration and enrichment}

Plasma FFA concentration and isotopic enrichment were measured by LC/MS (SIM mode) according to Persson and coworkers (Persson et al. 2010). MalonylCoA, LCACoA content and isotopic enrichment were measured according to Blachnio-Zabielska and coworkers (Blachnio-Zabielska et al. 2011). LCAC concentration and isotopic enrichment $\left({ }^{13} \mathrm{C}_{16}\right.$-carnitine) were measured according to Sun and coworkers (Sun et al. 2006) with minor modification. The ceramide content and isotopic enrichment were measured according to BlachnioZabielska and coworkers (Blachnio-Zabielska et al. 2012). The content and isotopic enrichment of DAG were measured according to Blachnio-Zabielska and coworkers (Blachnio-Zabielska et al. 2013). All measurements of lipid concentration and enrichment were performed using an Agilent 1290 Infinity UHPLC/Agilent 6460 Triple Quad (Agilent). Detailed description of the methods is presented in 'Extended materials and methods' section of the supplement.

\section{Protein and RNA isolation}

Total RNA and protein were isolated from muscle samples using NucleoSpin RNA/Protein isolation kit (MachereyNagel, Bethlehem, PA, USA), according to procedure by Bahn and coworkers (Bahn et al. 2008). RNA and proteins were separated on NucleoSpin columns according to manufacturer guidelines.

\section{Western blot}

Following target proteins were quantified using primary antibodies: GLUT4, FABP, CD36, FATP1, CPT1, ACC, ACS, SPT, PAP2B, Akt, pAktSer473, pAktThr308, AS160,

Published by Bioscientifica Ltd. 
AS160Thr642 and GAPDH. Values were normalized to GAPDH protein expression measured in parallel runs and expressed as fold changes over control group values. All chemicals and equipment used for immunoblotting were purchased from Bio-Rad.

\section{RT-PCR}

The RNA was reverse transcribed into cDNA using iScript cDNA Synthesis Kit (Bio-Rad) with oligo $(d T)_{18}$. Specific primers for the ACC, ACS, CD36, CPT1, FABPpm, FATP1, GLUT4, SPT and GAPDH were designed using the Beacon Designer Software. The housekeeping gene GAPDH was used as the reference gene for quantification. Quantitative real-time PCR was performed with SYBR Green Supermix Kit (Bio-Rad) using a Bio-Rad Chromo4 system.

\section{Acyl-coenzyme A synthetase (ACSs) activity}

Acyl-coenzyme A synthetase (ACSs) activity was assayed in muscle microsomes according to method by de Jong and coworkers (de Jong et al. 2007), with the use of radiolabeled $\left[9,10-{ }^{3} \mathrm{H}(\mathrm{N})\right]$-palmitate (American Radiolabeled Chemicals, Inc. Saint Louis, MO, USA). The 'Extended materials and methods' supplement contains methodology description for the isolation of muscle microsomes and ACS synthase activity assay.

\section{Phosphatidate phosphatase 2 (PAP2) activity}

Activity of PAP2 in skeletal muscle microsomes was measured according to Martin and coworkers (Martin et al. 1991), with the use of radiolabeled L-a-dioleoyl [oleoyl1-14C]-glycerolo-3-phosphate (American Radiolabeled Chemicals). The method is described in 'Extended materials and methods' section of the supplement.

\section{Oral glucose tolerance test (OGTT), insulin tolerance test (IPTT), plasma insulin and HOMA-IR}

The OGTT and IPTT tests were performed 3 days apart during the final week of the experiment. The food was withdrawn at 06:00h, and animals were fasted for $6 \mathrm{~h}$. For OGTT, animals received oral gavage of glucose at a dose of $3 \mathrm{~g} / \mathrm{kg}$. For IPTT, animals received intraperitoneal injection of insulin at dose of $0.75 \mathrm{U} / \mathrm{kg}$ body weight. Blood samples from tail vein were measured at given intervals by glucometer (AccuCheck, Roche). Plasma insulin was measured by ELISA (Rat/Mouse Insulin Millipore).
HOMA-IR index value was calculated according to Cacho and coworkers (Cacho et al. 2008).

\section{Principal component analysis, correlation analysis and statistical significance estimation}

Statistical significance between groups was determined using ANOVA with the Tukey honestly significant difference post-hoc test for unequal n-numbers. Significance level was set at $P<0.05$. PCA was performed using Statistica 10.0 software package as described earlier (Zabielski et al. 2014). To prevent an artificial increase in the PCA model strength, we excluded a majority of closely interdependent variables.

\section{Results}

\section{Whole-body insulin sensitivity}

HFD-fed animals developed IR, as evidenced by elevated fasting blood glucose (Supplementary Table 1), impaired glucose tolerance (Fig. 2A and B), reduced insulin responsiveness (Fig. 2C and D) and increased HOMA-IR index. Metformin normalized insulin-related parameters to control values.

\section{Skeletal muscle insulin sensitivity}

Induction of IR in HFD animals was accompanied by decreased insulin-stimulated Akt phosphorylation (at Ser475 and Thr308) and AS160 phosphorylation in skeletal muscle (Fig. 2E, F and G). Improvement of insulin sensitivity in HFD/Met group was associated with significantly increased phosphorylation of Akt and AS160, as compared to HFD animals $(P<0.05)$. Expression of GLUT4 protein increased significantly in HFD/Met compared to all other groups (in all cases, $P<0.05$, Fig. 2H). There were no significant changes in Glut4 mRNA (data not shown).

\section{Plasma FFA concentration, muscle expression of FA transporters, ACS activity, acyl-CoA content and FSR}

HFD increased total plasma FFA content (Fig. 3A, Supplementary Table 2) and turnover rate (Supplementary Fig. 1C and D) compared to control $(P<0.05$ in all cases). Metformin treatment normalized above variables except for total plasma FA turnover, which was still higher than control. Both HFD-treated

Published by Bioscientifica Ltd. 
A
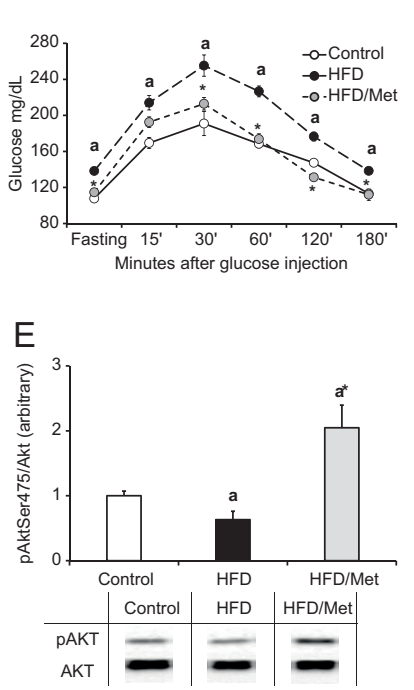

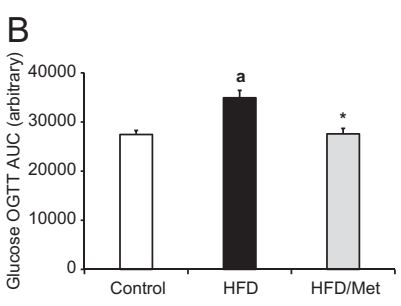

C

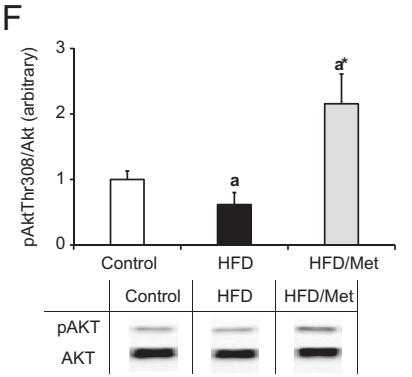

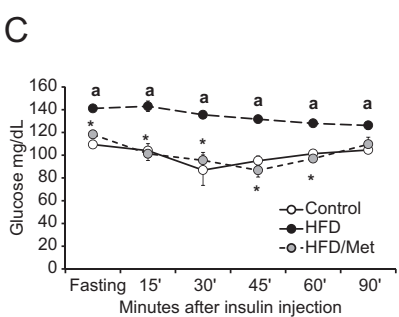
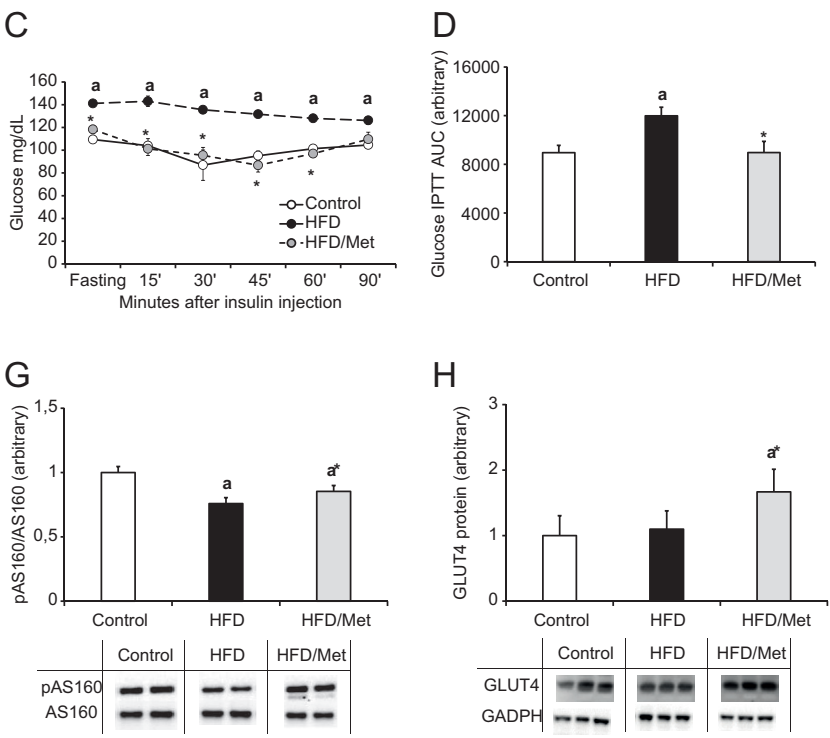

$\mathrm{H}$

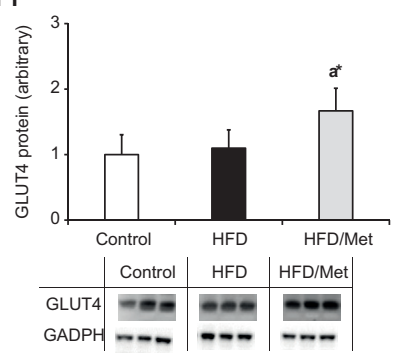

Figure 2

Metformin treatment improves glucose and insulin tolerance and muscle insulin signaling in HFD animals. Panels A and C - plasma glucose during OGTT and IPTT tests respectively. Panels B and D - plasma glucose area under curve (AUC) for OGTT and IPTT tests, respectively. Panel E and F - Akt/PKB phosphotylation at serine 473 and theonine 308, respectively; G and H - AS160 phosphorylation and GLUT4 expression, respectively. Values are mean \pm S.D. ( $n=8$ for each group), $P<0.05$ against: a- vs $C_{;}$*- vs HFD.

groups displayed significant increase in mRNA and protein levels of all measured fatty acid transporters (Fig. 3B and C). In the case of HFD/Met animals, the protein content of CD36 and FATP1 was the highest among all experimental groups. Increased plasma FFA and muscle FA transporters lead to accumulation of intramuscular LCACoA's in HFD group (Fig. 3D). Metformin strongly reduced the total LCACoA's in muscle, as compared to control and HFD animals. All of the above changes in the content of acyl-CoA were connected mainly with 18-carbon chain-derived acylCoAs (C18:0-, C18:1 and C18:2-CoA, Supplementary Table 3). Those species were responsible for more than $75 \%$ of the observed increase above control levels. Although LCACoA content varied significantly between experimental groups, the elevation in Acs mRNA (data not shown) and protein (Fig. 3E) were not significant. Enzymatic activity of ACS increased significantly in both HFD-treated groups (Fig. 3F). There was no significant variation in tissue C16:0-CoA enrichment or FSR between experimental groups (Supplementary Fig. 2A and $\mathrm{B}$ ), which indicates complete isotopic equilibration of acyl-CoA pool during $2 \mathrm{~h}$ of tracer infusion. This allowed for estimation of C16:0-CoA turnover rate, which significantly increased in HFD animals and returned to control values under metformin treatment (Supplementary Fig. 2C).

\section{Indicators of mitochondrial FA channeling}

Accumulation of LCACoA in muscle of HFD animals was accompanied by significantly decreased content and FSR of C16:0-AC (Fig. 4A, B and Supplementary Table 4) and Cpt1 mRNA expression (Fig. 4C). HFD significantly decreased $\mathrm{C} 16: 0-\mathrm{AC}$ to $\mathrm{C} 16: 0-\mathrm{CoA}$ ratio and respective FSR ratio (Fig. 4E and Supplementary Fig. 3), which suggest an inhibition of mitochondrial FA channeling. Metformin increased C16:0-AC content and FSR despite downregulation of CPT1 expression at both the mRNA and the protein level, as compared to control. Moreover, metformin increased C16:0-AC to C16:0-CoA ratio, and respective FSR ratios, which indicates augmentation of mitochondrial channeling of FA.

Muscle malonyl-CoA content doubled in HFD animals as compared to control (Fig. 4F). Although HFD decreased mRNA of Acc (Fig. 4G), the de-phosphorylation of ACC protein indicates its activation under HFD treatment (Fig. 4H). Metformin normalized ACC phosphorylation to control values and significantly decreased malonylCoA content.

\section{Muscle bioactive lipids}

HFD significantly increased muscle ceramide content (Fig. 5A), which was accompanied by the upregulation of

Published by Bioscientifica Ltd. 
A

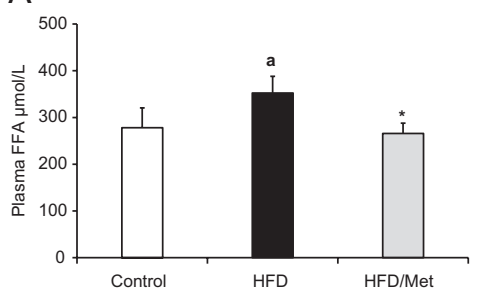

D

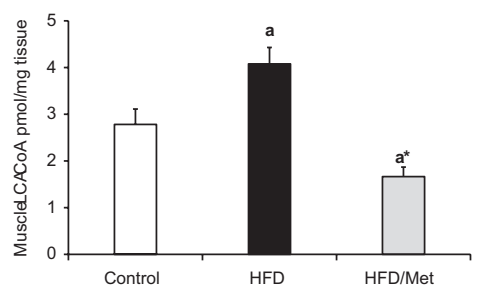

$\mathrm{B}$

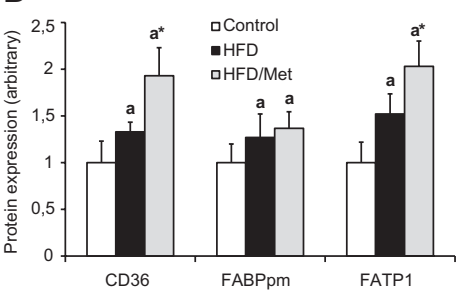

C
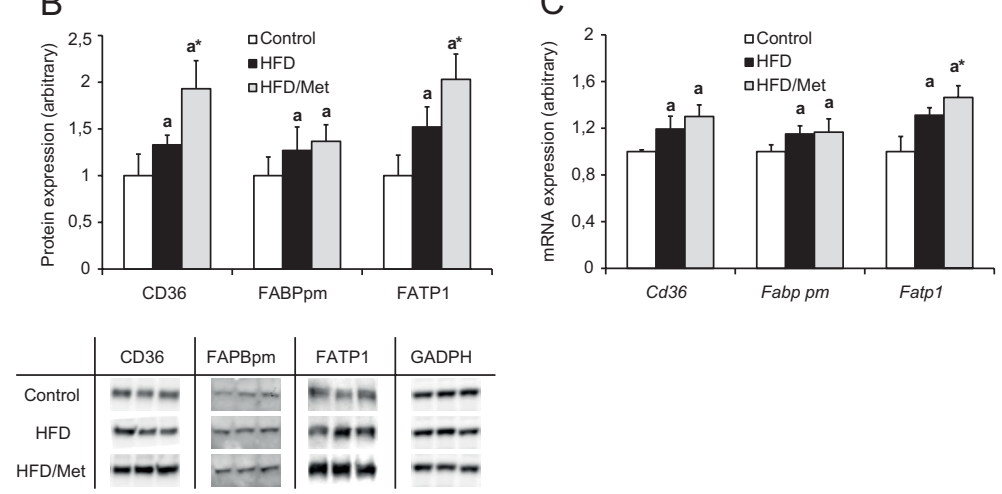

$\mathrm{E}$

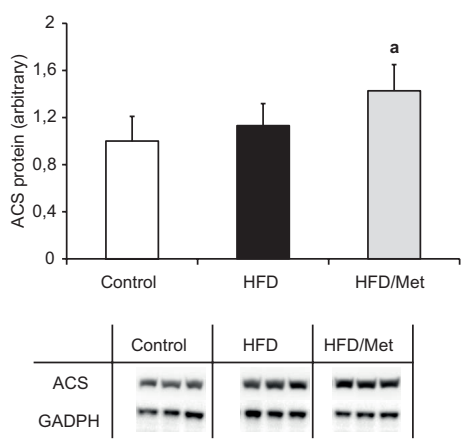

$\mathrm{F}$

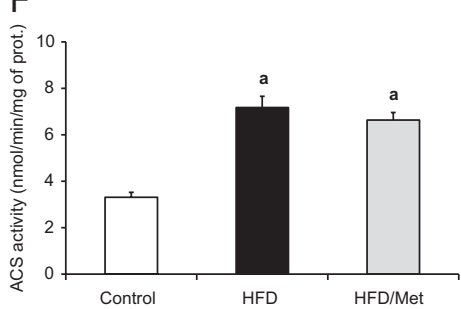

Figure 3

The effect of metformin on intracellular uptake and acyl-CoA conversion of fatty acids in in skeletal muscle of HFD-fed animals. Panel A - plasma FFA; B, C - protein and mRNA expression of Cd36, Fatp1 and Fabp pm. D - muscle acyl-CoA content; E and F - protein expression and enzymatic activity of ACS. Values are mean \pm S.D. ( $n=8$ for each group), $P<0.05$ against: a- vs $C$; *- vs HFD.

Spt mRNA and protein level (Fig. 5C, D and Supplementary Table 5). Interestingly, increase in the total content of Cer in HFD animals was not associated with an increase in FSR of C16:0-Cer (Fig. 5B). The C18:0 and C18:1 ceramides had the greatest impact on the total content of ceramide in HFD group (Supplementary Table 5 and accompanied figures). Both the FSR and content of C16:0-Cer did not increase despite HFD treatment, which indicates selective synthesis of ceramide species (Supplementary Fig. 4). Metformin normalized the total content of ceramide as well as mRNA and protein expression of SPT and decreased the FSR of 16:0-Cer.

High-fat diet triggered modest yet significant accumulation of intramuscular DAG (Fig. 5E), with C18:0/C18:2- and C18:2/C18:2-DAG accounting for majority of change in total DAG (Supplementary Table 6 and accompanied figures). Interestingly, as compared to control, the content of palmitate-derived DAG remained unchanged (16:0/16:0-DAG) or even decreased (16:0/18:1and 16:0/18:0-). The same could be noted for 16:0/16:0and 16:0/18:1-DAG FSR (Supplementary Fig. 5). The mismatch between total content of DAG and FSR of palmitate-derived DAGs suggests selective synthesis of 18-carbon acyl-chain DAGs under HFD treatment. Accumulation of DAG was accompanied by increased activity and protein expression of PAP2 (Fig. 5G and H). Metformin treatment decreased the total content of DAG, the FSR of 16:0/16:0- and 16:0/18:1-DAG, and both the activity and protein level of PAP2 ( $P<0.05$ vs HFD).

\section{Principal component (PCA) analysis}

Three major principal components were responsible for a total of $79 \%$ of cumulative variance within the data set $(\mathrm{pc} 1=45.8 \%, \mathrm{pc} 2=27.2 \%, \mathrm{pc} 3=6 \%$; cumulative $\mathrm{R} 2 \mathrm{X}=0.790, \mathrm{Q} 2=0.674)$. Scores biplot shows separate, tight clustering of animals from each experimental group (Fig. 6A), with clear separation of HFD group along t2 axis. Overlay of variable vectors show that factors that differentiate HFD group from control and HFD/Met animals were mostly indicators of insulin resistance and sensitivity, 18-carbon acyl-chain length Cer, DAG and acyl-CoA (along t2 axis). Also, variables associated with lipid mitochondrial channeling and insulin signaling, 
A

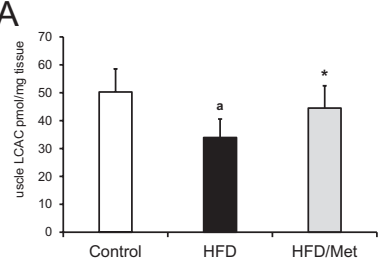

B

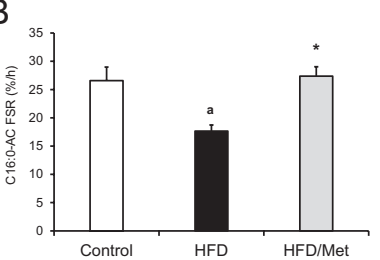

E

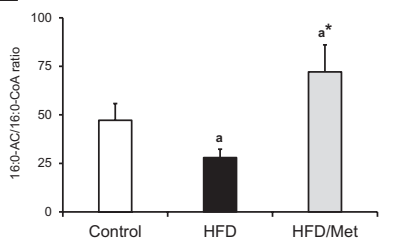

F

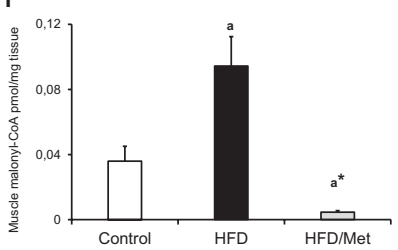

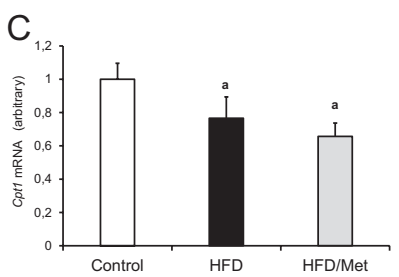

D

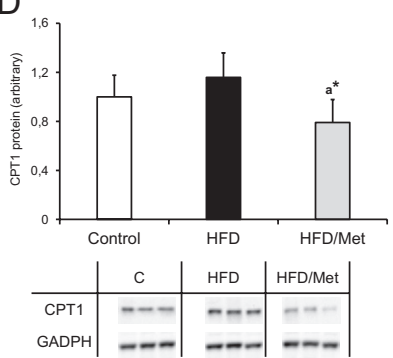

G

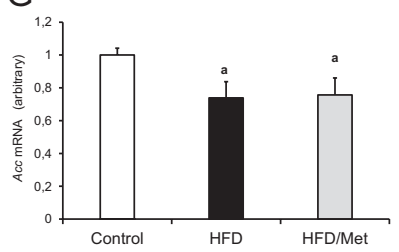

$\mathrm{H}$

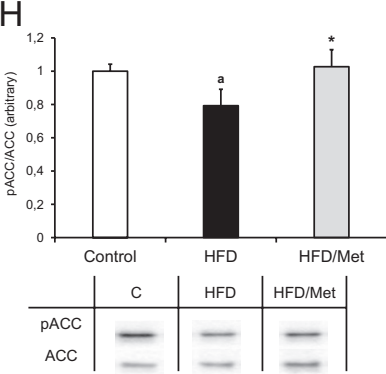

Figure 4

Metformin promotes mitochondrial channeling of fatty acids in skeletal muscle of HFD-fed animals. Panel A and B - total long-chain acyl-carnitine (LCAC) content and FSR; Panel C and D - mRNA and protein expression of CPT1. Panel E - 16:0-AC to 16:0-CoA ratio (a measure of mitochondrial fatty acids uptake); Panel F - malonyl-CoA content; Panels $\mathrm{G}$ and $\mathrm{H}-\mathrm{mRNA}$ expression and protein phosphorylation of ACC. Values are mean \pm S.D. $(n=8$ for each group), $P<0.05$ against: a- vs $C$; *- vs HFD.

such as total acyl-carnitine content and FSR, acylcarnitine to acyl-CoA ratio and Akt phosphorylation distinguished control and HFD/Met group from HFD
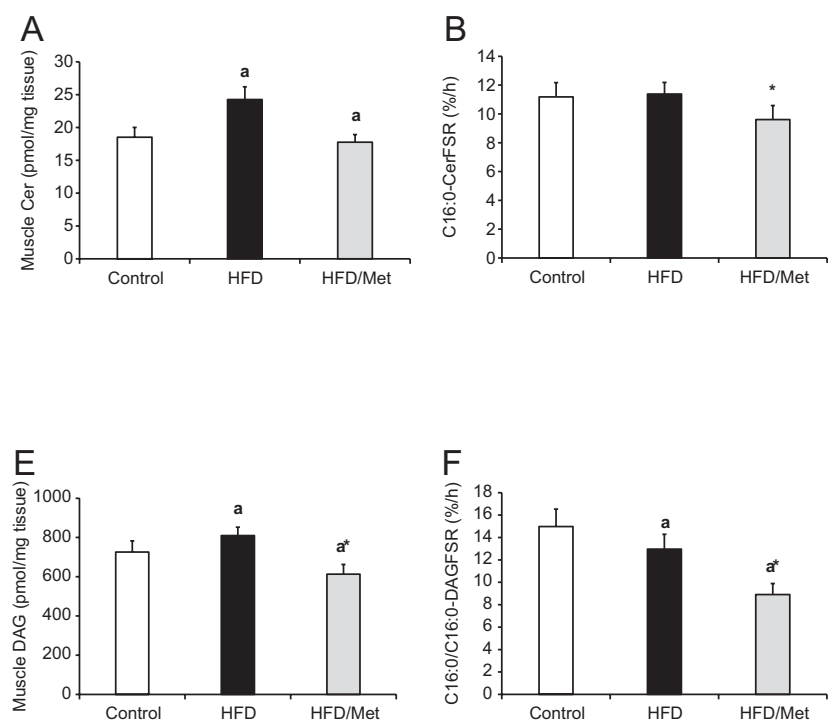

animals. Surprisingly, some of the Cer and all palmitatederived DAGs were associated with the control animals, suggesting that those lipid species are not involved in the
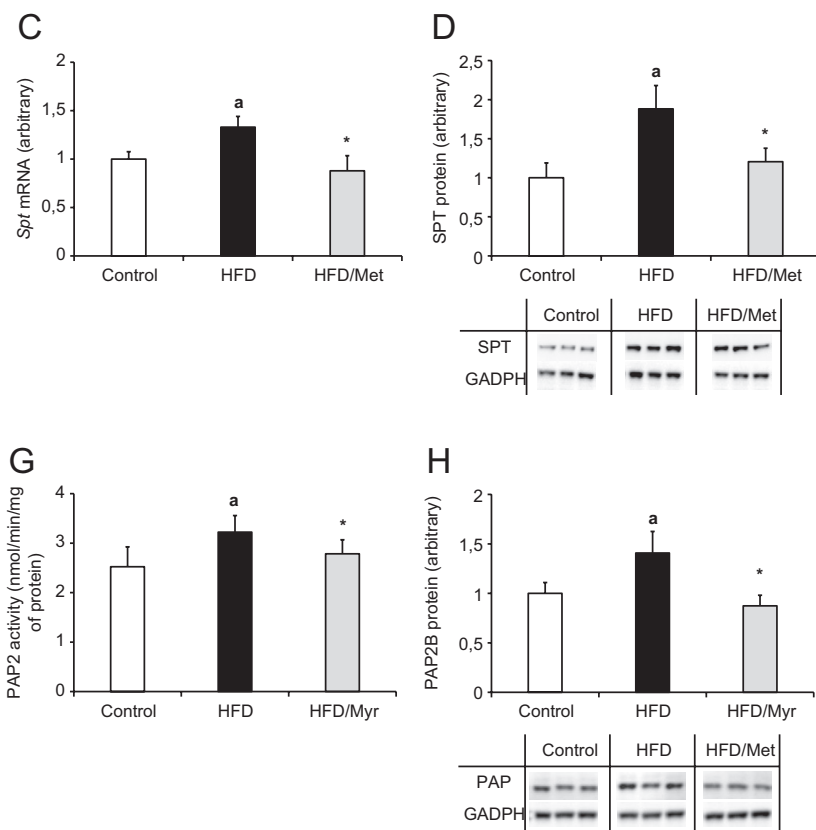

Figure 5

Metformin inhibits synthesis of Cer and DAG in skeletal muscle of HFD-fed animals. Panels A and B - total ceramide content and C16:0-Cer FSR; Panels C and D mRNA and protein expression of SPT; Panels E and F-total DAG content 16:0/16:0-DAG FSR; Panels G and H - phosphatidic acid phosphatase 2 (PAP2) activity and protein expression. Values are mean \pm s.D. $(n=8$ for each group), $P<0.05$ against: a- vs C; *- vs HFD.

http://joe.endocrinology-journals.org DOI: $10.1530 / \mathrm{JOE}-16-0381$ (c) 2017 Society for Endocrinology Printed in Great Britain
Published by Bioscientifica Ltd. 
A

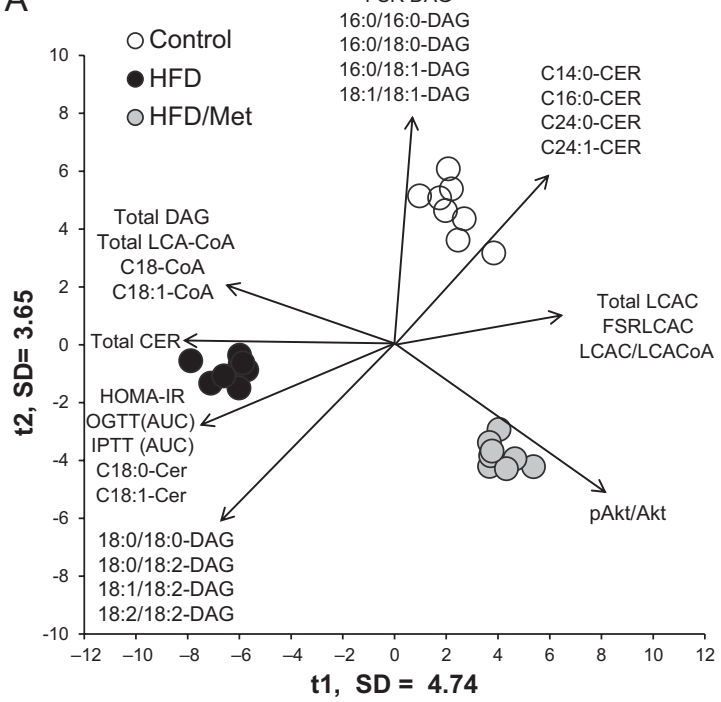

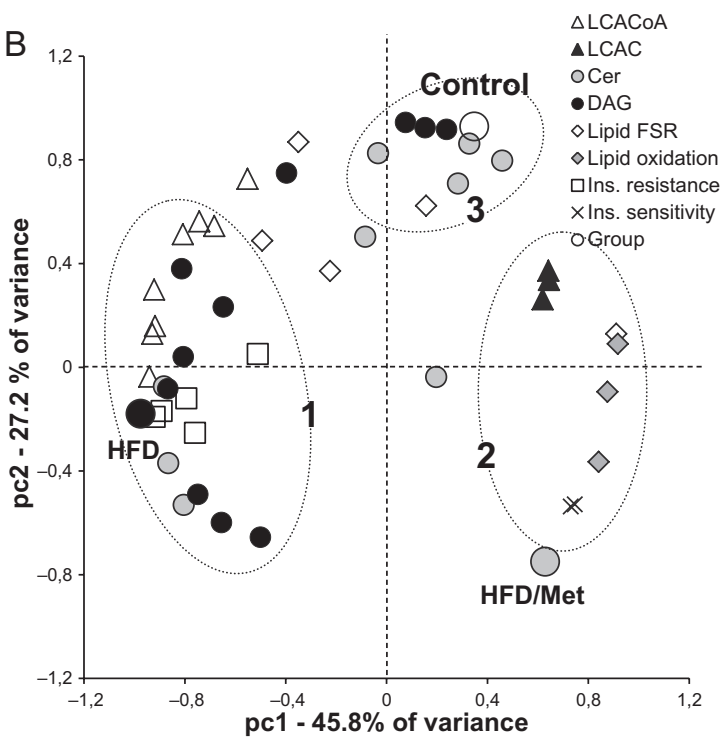

\section{Figure 6}

Principal component analysis (PCA) reveals association of 18-carbon acyl-chain length Cer and DAG with insulin resistance measures in HFD-fed animals. Panel A - scores scatter plot for 1st and 2nd PCA components. Arrows and names indicate direction and type and of major variables responsible for group differences. Panel B - loadings scatter plot for 2 first PCA components. The variables grouped in 3 major clusters (encircled) are described in 'Results' section.

induction of IR. Loadings scatter plot revealed clustering of both the 18-carbon acyl-chain Cers and acyl-CoAs with the indicators of IR such as HOMA-IR, fasting insulin and glucose, OGTT and IPTT AUC (Feature cluster 1, Fig. 6B). Other cluster members were malonyl-CoA, 18-carbon acyl-chain and total DAG content (except di-oleoylDAG). Variables related to FA mitochondrial channeling clustered together with HFD/Met group, acyl-carnitine content and FSR and Akt phosphorylation state (Feature cluster 2, Fig. 6B).

\section{Discussion}

In skeletal muscle, IR has been linked to bioactive lipid accumulation such as LCACoA, DAG and Cer (Pan et al. 1997, Cooney et al. 2002, Itani et al. 2002, Bonen et al. 2004, Powell et al. 2004, Straczkowski et al. 2007). It has been postulated that accumulation of these lipid intermediates induces defects in the insulin signaling cascade (Houmard et al. 2002, Bruce et al. 2006) and decreases insulin-stimulated glucose uptake (Hegarty et al. 2003). Metformin, a commonly used hypoglycemic drug, lowers blood glucose by decreasing hepatic glucose production and increasing glucose uptake by skeletal muscle. However, the relationship between insulinsensitizing effects of metformin and bioactive lipids is not completely understood. Our study was designed to evaluate the involvement of particular lipid species in induction of IR and to evaluate the impact of metformin on muscle bioactive lipid metabolism. We employed a stable isotope-labeled palmitate tracer and MS-based methods to measure muscle concentration and synthesis rates of palmitate-derived lipids that are known inhibitors of insulin signaling pathway (LCACoA, DAG and Cer).

High rates of FA uptake into skeletal muscle may, at least in part, be responsible for IMCL accumulation. Previously, it has been shown that muscle of obese or T2D animals display increased rates of FA uptake (Luiken et al. 2001). Our results indicate that skeletal muscle IR in HFD animals is associated with increased content and turnover rate of plasma FFA and skeletal muscle LCACoA due to upregulation in FA transporters and ACS activity. LCACoA are oxidized in mitochondria or used in lipid de novo synthesis. In our study, we tried to answer the question of how HFD consumption affects LCACoA channeling and what is the impact of metformin on this process. Mitochondrial $\beta$-oxidation is dependent on activity of carnitine palmitoyltransferase 1 (CPT1) and intracellular level of CPT1 inhibitor malonyl-CoA (Drynan et al. 1996). Malonyl-CoA is synthesized from acetyl-CoA by the action of acetyl-CoA carboxylase (ACC) (McGarry et al. 1983). ACC is inhibited by AMPK-dependent phosphorylation. It has been found that skeletal muscle accumulates malonyl-CoA under hyperglycemia and hyperinsulinemia (Sidossis et al. 1999, Rasmussen et al. 2002), which are

Published by Bioscientifica Ltd. 
associated with IR. It is suggested that the re-channeling of FA from oxidation to IMCL synthesis observed with IR may be attributable to high malonyl-CoA levels and high ACC activity. In our study, HFD consumption triggered accumulation of intramyocellular malonylCoA and activation of ACC, which was accompanied by decreased acyl-carnitine content, fractional synthesis rate and acyl-carnitine to acyl-CoA ratio. Our results indicate that in insulin-resistant muscle, there is a mismatch between fatty acid uptake and mitochondrial channeling, which leads to the accumulation of LCACoA. Metformin augmented mitochondrial channeling of FA as indicated by the decrease in content and turnover rate of plasma FFA and muscular LCACoA. Moreover, we observed a significant decline in malonoyl-CoA and inhibition of ACC, accompanied by increased content and FSR of acyl-carnitine, acyl-carnitine/acyl-CoA ratio and their respective FSR ratio as compared to HFD group. All observed changes suggest increased mitochondrial channeling of LCACoA under metformin treatment, which shifts fatty acids flux from IMCL synthesis toward $\beta$-oxidation.

HFD feeding led to the accumulation of muscular ceramide. However, not all Cer species were affected equally. Accumulation was most pronounced for C18:0Cer and C18:1-Cer. Interestingly, content and FSR of C16:0-Cer was not higher than control, suggesting selective synthesis of Cer species in skeletal muscle under HFD feeding. Although accumulation of C16:0-Cer was previously associated with hepatic insulin resistance (Raichur et al. 2014, Turpin et al. 2014), there is a growing body of evidence that 18-carbon fatty acyl-chain ceramides associate with skeletal muscle IR. Those Cer species are responsible for majority of Cer accumulation in skeletal muscle of HFD-fed animals (Lanza et al. 2013), mice with uncontrolled STZ-diabetes (Zabielski et al. 2014) as well as in obese, type 2 diabetic patients (Bergman et al. 2016). Selective Cer synthesis also explains the mismatch between total ceramide accumulation and C16:0-Cer FSR observed in our study. We infused labeled palmitate to track synthesis of Cer, whereas stearoyl and oleoyl ceramides were used to determine the total content of intramuscular Cer. The improvement of skeletal muscle insulin sensitivity under metformin treatment was also associated with reduced 18-carbon FA-derived ceramides, whereas other ceramide species remained unaffected. The importance of those Cer species in HFD-induced skeletal muscle IR is further supported by the results of PCA analysis, which show close association of stearoyland oleoyl-Cer with the HFD group and the measures of muscular IR. Muscle DAG accumulation was evident in insulin resistance states and obesity (Turinsky et al. 1990, Oakes et al. 1994, Schmitz-Peiffer et al. 1997). Our data indicate that 18-carbon acyl-chain length DAGs account for majority of the increase in total DAG under HFD treatment. Among diacylglycerol species, 18:0/18:2- and 18:2/18:2-DAG displayed the strongest variation in content with both induction of muscular IR and improvement of IR under metformin treatment. In the study by Szendoredi and coworkers, those particular DAG species displayed the strongest negative correlation with glucose disposal rate and positive correlation with PKC- $\theta$ activation in insulin-resistant muscle of obese or T2D diabetic subjects (Szendroedi et al. 2014). Insulin resistance induced by lipid infusion in sedentary subjects is also connected with oleoyl-, linoloyl- and linoleoyl-DAG accumulation in skeletal muscle (Chow et al. 2014). Our findings indicate that 18-carbon acyl-chain DAGs are associated with the induction of skeletal muscle IR. Interestingly, the palmitate-derived DAGs do not accumulate in IR muscle due to decreased FSR, which suggests less impact of those DAG species on muscular insulin sensitivity. Although the results of FSR measurement of palmitate-derived lipids indicate selective synthesis of intramuscular lipid species, the definitive confirmation requires the use of multiple fatty acids tracers. The drawback of our study was the use of single-tracer approach. Although it allowed us for differentiation between palmitate-derived and other lipid species, we could not measure FSR of stearate-derived lipids. Stearate-derived lipids had the greatest impact on total intramuscular lipid content in each of the measured lipid classes, and future studies must include additional fatty acids tracers, which reflect the lipid composition of the tissues of interest. At the molecular level, DAGs stimulate serine/threonine kinases, which inhibit IRS-1 activation due to phosphorylation of other than tyrosine amino acid residues (Itani et al. 2002). Ceramide activates PP2A phosphatase, which decreases Akt phosphorylation (Adams et al. 2004, Hsieh et al. 2014). Both mechanisms lead to the inhibition of insulin signaling cascade, decrease in AS160 phosphorylation, GLUT4 translocation, reduction of glucose uptake and induction of IR (Turinsky et al. 1990, Adams et al. 2004, Holland et al. 2007). Previous studies showed that metformin increased glucose uptake in cultured human muscle cells (Sarabia et al. 1992) or muscle strips from diabetic subjects (Galuska et al. 1991) and prevents IR (Holland et al. 2007, Ussher et al. 2010); yet, there was no compelling evidence of involvement of particular lipid species in the processes. Our results indicate that decreased 18-carbon

Published by Bioscientifica Ltd 
acyl-chain-derived bioactive lipids (both Cer and DAG) is strongly associated with insulin-sensitizing effects of metformin and augmentation of both the Akt and AS160 phosphorylation in skeletal muscle of HFD-treated animals. Yet, it should be noted that total content and 18-carbon acyl-chain-derived Cer, as compared to similar DAG species, demonstrate the strongest association with induction and reduction of insulin resistance.

Taking together, on the one hand, HFD consumption increases plasma concentration of FFA, and on the other hand, impairs mitochondrial channeling of FA in skeletal muscle through malonyl-CoA-mediated inhibition of CPT1. As a result, we have observed an accumulation of LCACoA, DAGs and Cers, which was most notable for lipid species acylated with 18-carbon chain length fatty acids. These changes were accompanied by decreased phosphorylation of Akt (Ser473 and Thr308) and AS160 and impaired whole body glucose disposal. Metformin treatment augmented mitochondrial channeling of FA by the reduction of CPT1 inhibition by malonyl-CoA and decreased 18-carbon acyl-chain-derived bioactive lipids. Our results indicate that accumulation of bioactive lipids acylated with stearoyl and oleoyl and linoleoyl fatty acids plays a major role in the induction of skeletal insulin resistance.

\section{Supplementary data}

This is linked to the online version of the paper at http://dx.doi.org/10.1530/ JOE-16-0381.

\section{Declaration of interest}

The authors declare that there is no conflict of interest that could be perceived as prejudicing the impartiality of the research reported.

\section{Funding}

This work was supported by Foundation for Polish Science Grant HOMING PLUS/2010-2/1 and by the Medical University of Bialystok (Grants 14318558, 143-18559 and 143-18726).

\section{Author contribution statement}

A B Z conceived and designed the study; $P Z$ and $M C$ performed animal experiments and $A C S$ activity measurements; $A B Z$ performed all lipidrelated measurements; A B Z performed gene expression measurements; $P$ Z and $K$ C performed WB analysis; $M$ B performed hormone measurements; $P Z$ performed statistical and PCA analysis of the data; A B $Z$ and $P Z$ analyzed data and interpreted results of the experiments; $A B Z$ and $P Z$ prepared figures and drafted the manuscript; $A B Z$ and $J G$ edited and revised the manuscript and $A B Z$ coordinated and directed the project.

\section{Acknowledgements}

The authors thank Dr lan Lanza (Mayo Clinic, Rochester, MN, USA) for his support in improving the final draft of the manuscript.

\section{References}

Adams JM, Pratipanawatr T, Berria R, Wang E, DeFronzo RA, Sullards MC \& Mandarino LJ 2004 Ceramide content is increased in skeletal muscle from obese insulin-resistant humans. Diabetes 53 25-31. (doi:10.2337/diabetes.53.1.25)

Bahn A, Hagos Y, Reuter S, Balen D, Brzica H, Krick W, Burckhardt BC, Sabolic I \& Burckhardt G 2008 Identification of a new urate and high affinity nicotinate transporter, hOAT10 (SLC22A13). Journal of Biological Chemistry 283 16332-16341. (doi:10.1074/jbc.M800737200)

Belfort R, Mandarino L, Kashyap S, Wirfel K, Pratipanawatr T, Berria R, Defronzo RA \& Cusi K 2005 Dose-response effect of elevated plasma free fatty acid on insulin signaling. Diabetes 54 1640-1648. (doi:10.2337/diabetes.54.6.1640)

Bergman BC, Brozinick JT, Strauss A, Bacon S, Kerege A, Bui HH, Sanders P, Siddall P, Wei T, Thomas MK, et al. 2016 Muscle sphingolipids during rest and exercise: a C18:0 signature for insulin resistance in humans. Diabetologia 59 785-798. (doi:10.1007/s00125-015-3850-y)

Blachnio-Zabielska AU, Koutsari C \& Jensen MD 2011 Measuring longchain acyl-coenzyme A concentrations and enrichment using liquid chromatography/tandem mass spectrometry with selected reaction monitoring. Rapid Communications in Mass Spectrometry 25 2223-2230. (doi:10.1002/rcm.5110)

Blachnio-Zabielska AU, Persson XM, Koutsari C, Zabielski P \& Jensen MD 2012 A liquid chromatography/tandem mass spectrometry method for measuring the in vivo incorporation of plasma free fatty acids into intramyocellular ceramides in humans. Rapid Communications in Mass Spectrometry 26 1134-1140. (doi:10.1002/rcm.6216)

Blachnio-Zabielska AU, Zabielski P \& Jensen MD 2013 Intramyocellular diacylglycerol concentrations and [U-13C]palmitate isotopic enrichment measured by LC/MS/MS. Journal of Lipid Research $\mathbf{5 4}$ 1705-1711. (doi:10.1194/jlr.D035006)

Bonen A, Parolin ML, Steinberg GR, Calles-Escandon J, Tandon NN, Glatz JF, Luiken JJ, Heigenhauser GJ \& Dyck DJ 2004 Triacylglycerol accumulation in human obesity and type 2 diabetes is associated with increased rates of skeletal muscle fatty acid transport and increased sarcolemmal FAT/CD36. FASEB Journal 18 1144-1146. (doi:10.1096/ fj.03-1065fje)

Bruce CR, Anderson MJ, Carey AL, Newman DG, Bonen A, Kriketos AD, Cooney GJ \& Hawley JA 2003 Muscle oxidative capacity is a better predictor of insulin sensitivity than lipid status. Journal of Clinical Endocrinology and Metabolism 88 5444-5451. (doi:10.1210/jc.2003030791)

Bruce CR, Thrush AB, Mertz VA, Bezaire V, Chabowski A, Heigenhauser GJ \& Dyck DJ 2006 Endurance training in obese humans improves glucose tolerance and mitochondrial fatty acid oxidation and alters muscle lipid content. American Journal of Physiology: Endocrinology and Metabolism 291 E99-E107. (doi:10.1152/ajpendo.00587.2005)

Cacho J, Sevillano J, de Castro J, Herrera E \& Ramos MP 2008 Validation of simple indexes to assess insulin sensitivity during pregnancy in Wistar and Sprague-Dawley rats. American Journal of Physiology: Endocrinology and Metabolism 295 E1269-E1276. (doi:10.1152/ ajpendo.90207.2008)

Chow LS, Mashek DG, Austin E, Eberly LE, Persson XM, Mashek MT, Seaquist ER \& Jensen MD 2014 Training status diverges muscle diacylglycerol accumulation during free fatty acid elevation. American Journal of Physiology: Endocrinology and Metabolism 307 E124-E131. (doi:10.1152/ajpendo.00166.2014)

Cooney GJ, Thompson AL, Furler SM, Ye J \& Kraegen EW 2002 Muscle long-chain acyl CoA esters and insulin resistance. Annals of the New 
York Academy of Sciences 967 196-207. (doi:10.1111/j.1749-6632.2002. tb04276.x)

de Jong H, Neal AC, Coleman RA \& Lewin TM 2007 Ontogeny of mRNA expression and activity of long-chain acyl-CoA synthetase (ACSL) isoforms in Mus musculus heart. Biochimica et Biophysica Acta 1771 75-82. (doi:10.1016/j.bbalip.2006.11.007)

Drynan L, Quant PA \& Zammit VA 1996 Flux control exerted by mitochondrial outer membrane carnitine palmitoyltransferase over beta-oxidation, ketogenesis and tricarboxylic acid cycle activity in hepatocytes isolated from rats in different metabolic states. Biochemical Journal 317 791-795. (doi:10.1042/bj3170791)

Ellis BA, Poynten A, Lowy AJ, Furler SM, Chisholm DJ, Kraegen EW \& Cooney GJ 2000 Long-chain acyl-CoA esters as indicators of lipid metabolism and insulin sensitivity in rat and human muscle. American Journal of Physiology: Endocrinology and Metabolism 279 E554-E560. (doi:10.1016/0005-2760(70)90186-4)

Galuska D, Zierath J, Thörne A, Sonnenfeld T \& Wallberg-Henriksson H 1991 Metformin increases insulin-stimulated glucose transport in insulin-resistant human skeletal muscle. Diabetes and Metabolism 17 159-163.

Guo Z, Nielsen S, Burguera B \& Jensen MD 1997 Free fatty acid turnover measured using ultralow doses of [U-13C]palmitate. Journal of Lipid Research 38 1888-1895.

Hegarty BD, Furler SM, Ye J, Cooney GJ \& Kraegen EW 2003 The role of intramuscular lipid in insulin resistance. Acta Physiologica Scandinavica 178 373-383. (doi:10.1046/j.1365-201X.2003.01162.x)

Holland WL, Brozinick JT, Wang LP, Hawkins ED, Sargent KM, Liu Y, Narra K, Hoehn KL, Knotts TA, Siesky A, et al. 2007 Inhibition of ceramide synthesis ameliorates glucocorticoid-, saturated-fat-, and obesity-induced insulin resistance. Cell Metabolism 5 167-179. (doi:10.1016/j.cmet.2007.01.002)

Houmard JA, Tanner CJ, Yu C, Cunningham PG, Pories WJ, MacDonald KG \& Shulman GI 2002 Effect of weight loss on insulin sensitivity and intramuscular long-chain fatty acyl-CoAs in morbidly obese subjects. Diabetes 51 2959-2963. (doi:10.2337/diabetes.51.10.2959)

Hsieh CT, Chuang JH, Yang WC, Yin Y \& Lin Y 2014 Ceramide inhibits insulin-stimulated Akt phosphorylation through activation of Rheb/ mTORC1/S6K signaling in skeletal muscle. Cellular Signalling 26 1400-1408. (doi:10.1016/j.cellsig.2014.03.004)

Hulver MW, Berggren JR, Cortright RN, Dudek RW, Thompson RP, Pories WJ, MacDonald KG, Cline GW, Shulman GI, Dohm GL, et al. 2003 Skeletal muscle lipid metabolism with obesity. American Journal of Physiology: Endocrinology and Metabolism 284 E741-E747. (doi:10.1152/ajpendo.00514.2002)

Itani SI, Ruderman NB, Schmieder F \& Boden G 2002 Lipid-induced insulin resistance in human muscle is associated with changes in diacylglycerol, protein kinase C, and IkappaB-alpha. Diabetes 51 2005-2011. (doi:10.2337/diabetes.51.7.2005)

Jeukendrup AE 2002 Regulation of fat metabolism in skeletal muscle. Annals of the New York Academy of Sciences 967 217-235. (doi:10.1111/j.1749-6632.2002.tb04278.x)

Kitessa SM \& Abeywardena MY 2016 Lipid-induced insulin resistance in skeletal muscle: the chase for the culprit goes from total intramuscular fat to lipid intermediates, and finally to species of lipid intermediates. Nutrients 8 466. (doi:10.3390/nu8080466)

Lanza IR, Blachnio-Zabielska A, Johnson ML, Coenen-Schimke JM, Jakaitis DR, Lebrasseur NK, Jensen MD, Nair KS \& Zabielski P 2013 Influence of fish oil on skeletal muscle mitochondrial energetics and lipid metabolites during high-fat diet. American Journal of Physiology: Endocrinology and Metabolism 304 E1391-E1403. (doi:10.1152/ ajpendo.00584.2012)

Luiken JJ, Arumugam Y, Dyck DJ, Bell RC, Pelsers MM, Turcotte LP, Tandon NN, Glatz JF \& Bonen A 2001 Increased rates of fatty acid uptake and plasmalemmal fatty acid transporters in obese Zucker rats. Journal of Biological Chemistry 276 40567-40573. (doi:10.1074/jbc. M100052200)
Martin A, Gomez-Munoz A, Jamal Z \& Brindley DN 1991 Characterization and assay of phosphatidate phosphatase. Methods in Enzymology 197 553-563. (doi:10.1016/0076-6879(91)97183-y)

McGarry JD, Mills SE, Long CS \& Foster DW 1983 Observations on the affinity for carnitine, and malonyl-CoA sensitivity, of carnitine palmitoyltransferase I in animal and human tissues. Demonstration of the presence of malonyl-CoA in non-hepatic tissues of the rat. Biochemical Journal 214 21-28. (doi:10.1042/bj2140021)

Oakes ND, Kennedy CJ, Jenkins AB, Laybutt DR, Chisholm DJ \& Kraegen EW 1994 A new antidiabetic agent, BRL 49653, reduces lipid availability and improves insulin action and glucoregulation in the rat. Diabetes 43 1203-1210. (doi:10.2337/diab.43.10.1203)

Pan DA, Lillioja S, Kriketos AD, Milner MR, Baur LA, Bogardus C, Jenkins AB \& Storlien LH 1997 Skeletal muscle triglyceride levels are inversely related to insulin action. Diabetes 46 983-988. (doi:10.2337/ diab.46.6.983)

Persson XM, Blachnio-Zabielska AU \& Jensen MD 2010 Rapid measurement of plasma free fatty acid concentration and isotopic enrichment using LC/MS. Journal of Lipid Research 51 2761-2765. (doi:10.1194/jlr.M008011)

Powell DJ, Turban S, Gray A, Hajduch E \& Hundal HS 2004 Intracellular ceramide synthesis and protein kinase Czeta activation play an essential role in palmitate-induced insulin resistance in rat L6 skeletal muscle cells. Biochemical Journal 382 619-629. (doi:10.1042/ BJ20040139)

Raichur S, Wang ST, Chan PW, Li Y, Ching J, Chaurasia B, Dogra S, Ohman MK, Takeda K, Sugii S, et al. 2014 CerS2 haploinsufficiency inhibits beta-oxidation and confers susceptibility to diet-induced steatohepatitis and insulin resistance. Cell Metabolism 20 687-695. (doi:10.1016/j.cmet.2014.09.015)

Rasmussen BB, Holmbäck UC, Volpi E, Morio-Liondore B, Paddon-Jones D \& Wolfe RR 2002 Malonyl coenzyme A and the regulation of functional carnitine palmitoyltransferase- 1 activity and fat oxidation in human skeletal muscle. Journal of Clinical Investigation 110 1687-1693. (doi:10.1172/JCI0215715)

Roepstorff C, Helge JW, Vistisen B \& Kiens B 2004 Studies of plasma membrane fatty acid-binding protein and other lipid-binding proteins in human skeletal muscle. Proceedings of the Nutrition Society 63 239-244. (doi:10.1079/PNS2004332)

Sarabia V, Lam L, Burdett E, Leiter LA \& Klip A 1992 Glucose transport in human skeletal muscle cells in culture. Stimulation by insulin and metformin. Journal of Clinical Investigation 90 1386-1395. (doi:10.1172/JCI116005)

Schmitz-Peiffer C, Browne CL, Oakes ND, Watkinson A, Chisholm DJ, Kraegen EW \& Biden TJ 1997 Alterations in the expression and cellular localization of protein kinase $\mathrm{C}$ isozymes epsilon and theta are associated with insulin resistance in skeletal muscle of the highfat-fed rat. Diabetes 46 169-178. (doi:10.2337/diab.46.2.169)

Schmitz-Peiffer C, Craig DL \& Biden TJ 1999 Ceramide generation is sufficient to account for the inhibition of the insulin-stimulated РКВ pathway in C2C12 skeletal muscle cells pretreated with palmitate. Journal of Biological Chemistry 274 24202-24210. (doi:10.1074/ jbc.274.34.24202)

Sidossis LS, Mittendorfer B, Chinkes D, Walser E \& Wolfe RR 1999 Effect of hyperglycemia-hyperinsulinemia on whole body and regional fatty acid metabolism. American Journal of Physiology $\mathbf{2 7 6}$ E427-E434.

Simoneau JA, Veerkamp JH, Turcotte LP \& Kelley DE 1999 Markers of capacity to utilize fatty acids in human skeletal muscle: relation to insulin resistance and obesity and effects of weight loss. FASEB Journal 13 2051-2060.

Straczkowski M, Kowalska I, Baranowski M, Nikolajuk A, Otziomek E, Zabielski P, Adamska A, Blachnio A, Gorski J \& Gorska M 2007 Increased skeletal muscle ceramide level in men at risk of developing type 2 diabetes. Diabetologia 50 2366-2373. (doi:10.1007/s00125-0070781-2)

Published by Bioscientifica Ltd. 
Sun D, Cree MG, Zhang XJ, Bøersheim E \& Wolfe RR 2006 Measurement of stable isotopic enrichment and concentration of long-chain fatty acyl-carnitines in tissue by HPLC-MS. Journal of Lipid Research 47 431-439. (doi:10.1194/jlr.D500026-JLR200)

Szendroedi J, Yoshimura T, Phielix E, Koliaki C, Marcucci M, Zhang D, Jelenik T, Muller J, Herder C, Nowotny P, et al. 2014 Role of diacylglycerol activation of PKCtheta in lipid-induced muscle insulin resistance in humans. PNAS 111 9597-9602. (doi:10.1073/pnas.1409229111)

Turinsky J, O'Sullivan DM \& Bayly BP 1990 1,2-Diacylglycerol and ceramide levels in insulin-resistant tissues of the rat in vivo. Journal of Biological Chemistry 265 16880-16885.

Turpin SM, Nicholls HT, Willmes DM, Mourier A, Brodesser S, Wunderlich CM, Mauer J, Xu E, Hammerschmidt P, Bronneke HS, et al. 2014 Obesity-induced CerS6-dependent C16:0 ceramide production promotes weight gain and glucose intolerance. Cell Metabolism 20 678-686. (doi:10.1016/j.cmet.2014.08.002)

Ussher JR, Koves TR, Cadete VJ, Zhang L, Jaswal JS, Swyrd SJ, Lopaschuk DG, Proctor SD, Keung W, Muoio DM, et al. 2010 Inhibition of de novo ceramide synthesis reverses diet-induced insulin resistance and enhances whole-body oxygen consumption. Diabetes 59 2453-2464. (doi:10.2337/db09-1293)

Zabielski P, Blachnio-Zabielska A, Lanza IR, Gopala S, Manjunatha S, Jakaitis DR, Persson XM, Gransee J, Klaus KA, Schimke JM, et al. 2014 Impact of insulin deprivation and treatment on sphingolipid distribution in different muscle subcellular compartments of streptozotocin-diabetic C57Bl/6 mice. American Journal of Physiology: Endocrinology and Metabolism 306 E529-E542. (doi:10.1152/ ajpcell.00022.2014)

Received in final form 14 March 2017

Accepted 11 April 2017 (c) 2017 Society for Endocrinology Printed in Great Britain
Published by Bioscientifica Ltd. 